\title{
Using ALLiS for Clausing
}

\author{
Hervé Déjean \\ Seminar für Sprachwissenschaft \\ Universität Tübingen \\ de jean@sfs.nphil. uni-tuebingen.de
}

\begin{abstract}
We present the result of a symbolic machine learning system, ALLiS 2.0 for the CoNLL-2001 shared task. ALLiS 2.0 is a theory refinement system using hierarchical data. Results are $\mathrm{F}=89.04$ for subtask $1, \mathrm{~F}=68.02$ for subtask 2 and $\mathrm{F}=67.70$ for subtask 3 (development test). Adding manual rules improves considerably results specially for task $2(\mathrm{~F}=79.44)$. For the test data, results are slightly worst $(\mathrm{F}=62.27$ for subtask 3).
\end{abstract}

\section{Introduction}

ALLiS (Architecture for Learning Linguistic Structure) (Déjean, 2000a), (Déjean, 2000b) is a symbolic machine learning system. The learning system is based on theory refinement. It tries to refine (to improve) an existing imperfect grammar using operators such as contextualization and lexicalization. ALLiS separates the task of the generation of rules and the task of the use of these rules (task of parsing). First symbolic rules are learned and saved using an own formalism, and in a second time, these rules are converted into a proper formalism used by a specific rule-based parser. ALLiS uses XML formalism for learning as well as for parsing. The following XML Components are:

- LT TTT, a text tokenization system and toolset which enables users to produce a swift and individually-tailored tokenization of text.

- LT XML, an integrated set of XML tools and a developers tool-kit, including a Cbased API.

- XMLQUERY, (Mckelvie, 2000), an extension of the LTXML query language to allow more complex queries including operators for finding sequences of XML elements.

\section{Theory Refinement}

We present here a brief introduction to theory refinement. For a more detailed presentation, we refer the reader to (Abecker and Schmid, 1996), (Brunk, 1996) or (Mooney, 1993). (Mooney, 1993) defines it as:

Theory refinement systems developed in Machine Learning automatically modify a Knowledge Base to render it consistent with a set of classified training examples.

This technique thus consists of improving a given Knowledge Base (here a grammar) on the basis of examples (here a treebank). Some impose to modify the initial knowledge base as little as possible. Applied in conjunction with existing learning techniques (Explanation-Based Learning, Inductive Logic Programming), TR seems to achieve better results than these techniques used alone (Mooney, 1997). It consists of two main steps:

1. Build a more or less correct grammar on the basis of background knowledge.

2. Refine this grammar using training examples:

(a) Identify the revision points

(b) Correct them

The first step consists in acquiring an initial grammar (or more generally a knowledge base). In this work, the initial grammar is automatically induced from a tagged and bracketed corpus. The second step (the refinement) compares the prediction of the initial grammar with the training corpus in order to firstly identify the revision points, i.e. points that are not correctly described by the grammar, and secondly, to correct these revision points. 
If a NP with an NNP occurs between a PP containing IN/for and a VP containg $\mathrm{TO} /$ to, it is a start of clause.

\section{Rules}

For data test1, ALLiS has learned 149 rules, the coverage of the learning test being $82 \%$ ( $82 \%$ of the positive cases are explained). As usually, few very frequent rules insure the main part of the coverage.

Concerning the second data, rules are more difficult to learn. ALLiS only generates 25 rules and the recall is very low.

\subsection{Which precision?}

Another important parameter is the threshold $\theta$ which determines when the accuracy of a rule is high enough. We tried two values: 0.8 and 0.9 . For the chunking task (last year sharedtask), the best value was 0.9 . In this task, the best one is 0.8 . We think that the explanation is partially due to a more important quatity of noise in data. For the chunking task, the noise was due to POS tagging. In this data, it is due to errors of tagging and also errors of chunking. The F-score being around $92 \%$ for chunking, it is obvious that the error rate for some tags is higher than $10 \%$. We can note that, even if the threshold is set up at 0.8 , the overall precision stays high enough $(94.08 \%$ against $95.76 \%$ for $\theta=0.9$ ). Furthermore, setting $\theta$ at 0.8 allows a better recall $(84.59 \%$ against $78.58 \%)$. It then seems that $\theta$ 's value should be correlated with the estimation of the noise in data.

\section{Result}

For CoNLL'01 shared-task 1, ALLiS offers a good precision but a lower recall. Using a window of one element before and after seems to be sufficient. Concerning subtask 2, the result is unfortunately very close to the baseline. It is clear that this task does not need local information, but information about the existence of opened clauses. In order to validate this affirmation, we add a new rule which consists in adding a tag $B={ }^{\prime} E$ ' at the last word of the sentence when two clauses are open. This improves greatly recall (72.76) and precision keeps high enough (87.48) to improve the overall result $(\mathrm{F}=79.44)$.

Results of the task 3 are provided by combining task 1 and 2 and using a Perl script provided

\begin{tabular}{|l|c|c|c|}
\cline { 2 - 4 } \multicolumn{1}{c|}{ developement } & precision & recall & $\mathrm{F}_{\beta=1}$ \\
\hline part 1 & $94.08 \%$ & $84.59 \%$ & 89.08 \\
part 2 & $99.28 \%$ & $51.73 \%$ & 68.02 \\
part 3 & $73.93 \%$ & $62.44 \%$ & 67.70 \\
\hline
\end{tabular}

\begin{tabular}{|l|c|c|c|}
\cline { 2 - 4 } \multicolumn{1}{c|}{ test } & precision & recall & $\mathrm{F}_{\beta=1}$ \\
\hline part 1 & $93.76 \%$ & $81.90 \%$ & 87.43 \\
part 2 & $99.04 \%$ & $48.90 \%$ & 65.47 \\
part 3 & $72.56 \%$ & $54.55 \%$ & 62.27 \\
\hline
\end{tabular}

Table 1: ALLiS results

by Erik Tjong Kim Sang.

\section{References}

Andreas Abecker and Klaus Schmid. 1996. From theory refinement to $\mathrm{kb}$ maintenance: a position statement. In $E C A I^{\prime} 96$, Budapest, Hungary.

Clifford Alan Brunk. 1996. An investigation of Knowledge Intensive Approaches to Concept Learning and Theory Refinement. Ph.D. thesis, University of California, Irvine.

Hervé Déjean. 2000a. Theory refinement and natural language learning. In COLING'2000, Saarbrücken.

Hervé Déjean. 2000b. A use of $\mathrm{xml}$ for machine learning. In Proceeding of the workshop on Computational Natural Language Learning, CONLL'2000.

David Mckelvie, 2000. XML QUERY 2.0. Edinburgh. http://www.ltg.ed.ac.uk/software/ttt/.

Raymond J. Mooney. 1993. Induction over the unexplained: Using overly-general domain theories to aid concept learning. Machine Learning, 10:79.

Raymond J. Mooney. 1997. Inductive logic programming for natural language processing. In Sixth International Inductive Logic Programming Workshop, pages 205-224, Stockholm,Sweden.

Erik F. Tjong Kim Sang and Hervé Déjean. 2001. Introduction to the conll-2001 shared task: Clause identification. In Proceedings of CoNLL, shared task. 